\title{
La secuencia de cine como técnica subjetiva en psicoterapia audiovisual
}

\author{
María Olga RuEDA CUENCA ${ }^{1}$ \\ psico@espaciointerno.net-olga.rueda@yahoo.es
}

Recibido: $15 / 03 / 12$

Aceptado: 22/11/12

\section{RESUMEN}

El presente trabajo es un resumen de un estudio basado en la aplicación de la Técnica de la Secuencia de Cine (TSC) como herramienta subjetiva, en clientes del Taller de Videoterapia Experimental, en "espaciointerno psicología" de Madrid.

El tipo de metodología utilizado en este estudio es descriptivo, de orientación cualitativa, mediante observación abierta participante. Se realizó una revisión del material filmico seleccionado, retro y prospectivamente, generando información narrativa subjetiva que esclareciera la experiencia de los participantes. Se concluye este estudio con un señalamiento de direcciones para futuras investigaciones.

Palabras clave: Cineterapia; videoterapia; psicología de los medios; concepto de sí mismo; representación mental.

\section{Referencia normalizada}

Rueda Cuenca, M. O. (2012). "La secuencia de cine como técnica subjetiva en psicoterapia audiovisual". En Arteterapia: Papeles de arteterapia y educación artística para la inclusión social Vol.7: páginas 189-206. Madrid. Servicio de publicaciones UCM.

\section{SUMARIO}

Introducción. El cine en terapia y la TSC. Método. Estrategias metodológicas. Población y muestra. Instrumentos de recogida de información. Técnica de la Secuencia de Cine (TSC). Anotaciones de la terapeuta-investigadora. Entrevista. Blog. Procedimiento. Resultados. Discusión y conclusiones. Referencia bibliográficas.

\section{The sequence of film as subjective technique in audiovisual psychotherapy}

\begin{abstract}
The present work is a summary of a study based on applying the Sequence Technique in Cinema (TSC) as a subjective tool with clients of the Experimental Videotherapy Workshop, at "espaciointerno psicología" in Madrid.

A descriptive methodology, of qualitative orientation, through an open participative observation has been used to elaborate this study. To be able to generate some subjective, narrative information with the power to clarify the experience of the participants, a retro and prospective review of the selected filmic material has been done. Hints for future investigations show themselves as being the conclusion of this study.
\end{abstract}

\footnotetext{
${ }^{1}$ Psicóloga y psicoterapeuta gestalt. Realizadora de televisión. Dirige espaciointerno psicología en donde desarrolla el Taller de Videoterapia Experimental.

Doctoranda en Dpto. Educación Artística, Plástica y Visual, Universidad Autónoma de Madrid, (UAM), España.
} 
Keywords: Cinematherapy; videotherapy; media psychology; self concept; mental representation.

\section{CONTENTS}

Introduction. The Cinema in Therapy and the TSC. The Method. Methodological strategies. Population and sample. Information gathering tools. Sequence Technique in Cinema (TSC). Interview. Blog. Procedure. Results. Discussion and conclusions. Bibliographic references.

\section{INTRODUCCIÓN}

Este trabajo se centra principalmente en la exploración del autoconcepto mediante metodologías audiovisuales. Con este objetivo nace en el año 2005 el Taller de Videoterapia Experimental con grupos de seis participantes. Ya se han realizado diez convocatorias, ocho de ellas válidas para este trabajo. Con el objeto de ir profundizando en nuevos contenidos, se siguen más de cincuenta ejercicios y consignas que varían de una sesión a otra. Son siete sesiones en total, con propuestas de videofeedback, videoconfrontación o de cineterapia, entre las que están "La técnica de la Secuencia de Cine o TSC" ${ }^{\circledR}{ }^{2}$ que se expone en este artículo y que se realiza en las sesiones 1 y 2 . El objetivo de las distintas técnicas es la descomposición funcional de los diferentes aspectos del autoconcepto, deconstruyéndolo en pequeños módulos de análisis para una mejor reapropiación de nuevos conceptos del sí mismo, más abiertos y ajustados. Se trata de un trabajo aplicado, enfocado como proceso de psicoterapia de apoyo con medios audiovisuales, por lo que las técnicas van orientadas a una intervención global. Es solo para la investigación que aislamos el estudio de cada técnica, como en este trabajo.

Dejando así apuntado el contexto en el que se desarrolla la "Técnica de la Secuencia de Cine" (en adelante TSC), mostraremos algunos de los resultados más sugerentes del trabajo de campo, que se ha apoyado en propuestas complementarias y narrativas según el modelo de Investigación Basada en las Artes, con un posicionamiento epistemológico acorde a los postulados de la psicoterapia constructivista.

La TSC podría considerarse una técnica subjetiva (Fernández-Ballesteros, 2001), en la que la fuente de datos es el propio sujeto que califica, clasifica o describe a sí mismo o a conceptos significativos, mediante material semiestructurado, no enmascarado, flexible y no tipificado y en las que la respuesta del sujeto es voluntaria.

Indagar el mundo interno implica tratar con el relato construido sobre sí mismo. No siempre consciente, el relato propio, aflora en cada una de nuestras manifestaciones. Mientras las técnicas proyectivas son una buena manera de expresar lo inconfesable incluso para nosotros mismos, este tipo de técnicas se apoyan en la dualidad científico/objeto-estudio y en la tipificación que facilitan la evaluación y diagnóstico. No obstante, las técnicas subjetivas presentan una mayor flexibilidad muy adecuada para la elicitación ${ }^{3}$ de constructos identitarios y para el análisis de significados personales en grupos de trabajo orientados al autoconocimiento.

\footnotetext{
${ }^{2}$ Taller de Videoterapia Experimental. Explorar el autoconcepto. Integrar la propia imagen” ${ }^{\circledR} \mathrm{n}^{\mathrm{o}}$ M-007498/2007

${ }^{3}$ Elicitar: anglicismo utilizado en psicología con el sentido que corresponde a los verbos españoles provocar, suscitar u obtener.
} 
Terapéuticamente no es suficiente con que el paciente exprese su mundo interior, es de gran importancia que pueda darse cuenta de qué manera su concepto de sí mismo es limitante o inoperante para su relación con los otros o con su contexto, porque solo de esa manera puede intervenir, él mismo, en su propio bienestar. Este es el propósito del Taller de Videoterapia Experimental y el de la técnica que aquí se presenta. El autoconcepto como representación mental que la persona tiene de sí misma, tanto a un nivel físico, como emocional e intelectual, es un marco de referencia necesario desde el que interpretar las propias experiencias y la realidad externa. En términos de la Psicología de los Constructos Personales, el self puede considerarse como una teoría personal compuesta de un conjunto de hipótesis sobre uno mismo, que mantienen y organizan el sentido de autoconsciencia en el tiempo (Botella y Feixas, 1998). Un autoconcepto, muy cerrado y rígido por ejemplo, puede ser tan inflexible que dificulte una adecuada adaptación al medio. Un buen ajuste del mismo contribuye a la salud y al equilibrio psíquico, permitiendo a una persona respetarse y valorarse tal como es y facilitando su relación con los demás.

Pensamos que cada individuo tiene la llave de su significado como ser. No solo el relato externo, biográfico, social, sino que el relato propio, oculto, no nombrado, contiene los elementos que propician las claves para estudiar lo personal y lo social. El sí mismo se construye sobre una base interdependiente de su contexto y coincidimos con Régis Debray (1994) en que cada época tiene un código figurativo que le impone como denominador común su arte dominante. Según la última encuesta realizada por el Ministerio de Educación y Ciencia respecto a los hábitos y prácticas culturales de los ciudadanos españoles ${ }^{4}$ podemos deducir que el visionado de películas supera en nuestro país a cualquier otra práctica cultural con diferencia. Aunque estemos inmersos en la cultura audiovisual que abarca cada vez más ramas entremezcladas de artes y saberes visuales y que parece acabar con el desarrollo lineal del discurso generando una identidad fragmentada en el individuo, el cine, como narrativa propia de los contadores de historias, aún pervive con fuerza en nuestra manera de aportar significados que se producen en nuestro contexto cultural. Por otro lado el cine es inseparable de la política cultural, y, como plantea Gi$\operatorname{roux}(2003)$ :

En el ámbito de la cultura, se forjan las identidades, se activan los derechos ciudadanos y se desarrollan las posibilidades de traducir actos de interpretación en formas de intervención y por tanto es necesario tener en cuenta la vinculación de la construcción del saber con cuestiones de ética, política y poder.

$Y$ en este sentido, la modesta investigación del presente estudio, señala la importancia de la industria norteamericana y sus modelos de identidad, para nuestra narrativa individual y colectiva (véase tabla 2 de resultados).

\footnotetext{
${ }^{4}$ Encuesta de hábitos y prácticas culturales de los ciudadanos españoles MEC 2006-2007
} 
Podemos tomar el cine como expresión social. Un lugar de encuentro con los múltiples mitos de la nueva épica de nuestro tiempo, una fuente de arquetipos que "todos" reconocemos y un lenguaje común - casi universal- expresión de nuestra manera actual de representar e interpretar el mundo. En él encontramos siempre un modelo en el que identificarnos bajo la perspectiva identitaria personal (Igartua, 2007). La variedad de relatos que ofrece el medio cinematográfico y lo extendido de su difusión hace del cine, por el momento, el arte de nuestro tiempo y un buen reflejo de "nuestros respectivos mundos".

\section{EL CINE EN TERAPIA Y LA TÉCNICA DE LA SECUENCIA DE CINE (TSC)}

Desde la Asociación de Videoterapia Española y Recursos Telemáticos (aVERTe), hemos iniciado una labor de indagación sobre las diferentes modalidades de aplicación de los medios audiovisuales en terapia (Mampaso, Rueda, Pérez, Morales, 2010). Dado lo novedoso del campo y la pluralidad de usos, nace la necesidad de definir cada ámbito de aplicación en función de los medios empleados. En ese trabajo intentamos una acotación de los posibles usos de los medios audiovisuales en terapia en general y del cine en particular. No es una labor fácil ya que las lindes tradicionales que se basaban en una distinción audiovisual según el soporte (quími$\mathrm{co}=$ cine; magnético $=$ vídeo), quedan rebasadas y confundidas por la diversidad de soportes actuales (químicos, ópticos, magnéticos y/o electrónicos), como el cine digital, el cine para tv, cine DVD, formatos para descarga en internet, etc.

Por tanto para simplificar, de manera provisional, sin mayores matices y por motivos didácticos, hemos incluido cualquier uso de material cinematográfico con soporte original fotoquímico ${ }^{5}$ destinado a fines terapéuticos, entre los que hemos definido el cineterapia, filmoterapia, cineforum terapéutico, cinearteterapia, cinefeedback, y los productos cinematográficos en torno a la terapia, que no describiremos aquí por problemas de extensión.

La TSC, objeto del presente trabajo, no es directiva o prescriptiva como en el caso de algunas de las aplicaciones nombradas en el párrafo anterior. El término con el que podríamos definir esta modalidad podría ser psico-secuencia. La característica diferenciadora de la TSC es precisamente la no intervención del terapeuta en la elección del material, frente a las propuestas conocidas como cinematherapy ${ }^{6}$ basadas en modelos prescriptivos que suponen que el experto indica el visionado de una película como intervención para el cambio. En la TSC, es la preferencia del partici-

\footnotetext{
${ }^{5}$ El soporte cinematográfico es una banda fabricada en acetato o poliéster transparente, sobre el que se adhieren varias capas o emulsiones de haluros de plata sensibles a la luz. Se trata de un soporte fotoquímico que solo ha de ser puesto a la luz en el momento de la exposición (en el momento de la toma de las imágenes) y que precisa un procesado en laboratorio para su visionado.

${ }^{6}$ Cinematherapy es el término acunado por los Hesley en 1998 en un betseller llamado Alquila un par de películas y nos vemos mañana, para brindar apoyo psicológico a los pacientes mediante el visionado de una película elegida por los terapeutas. Es amplia la bibliografía en relación a este método.
} 
pante la que guía el trabajo, quedando la figura del profesional en facilitador de la expresión genuina, ayudando a poner en conciencia, por medio de la verbalización y el darse cuenta de los significados del paciente, su propia estructura biográfica, emocional o identitaria.

Como explicábamos más arriba la TSC es una técnica subjetiva cuya fuente de datos es el propio sujeto, que mediante respuesta voluntaria escoge y describe una secuencia aislada de entre todas las películas que pueda recordar. Nos basamos en la premisa de que la selección de una secuencia estará motivada por el impacto emocional que los largometrajes pueden ejercer de manera incidental en los espectadores (Igartua, 2007). El motivo por el que preferimos una secuencia frente a una película, es por su fuerza narrativa concentrada. La película puede ir de esto o aquello, con un desfile de personajes, historias y subtemas que enriquecen el resultado global, pero de la que resulta difícil realizar una simplificación, sin embargo la secuencia es una unidad de narración en sí misma desarrollada en un tiempo muy breve y concentrado. Los participantes se convierten en "montadores" y buscan el comienzo y final de $\underline{s u}$ secuencia (a veces escogen una escena ${ }^{7}$ y es el propio sujeto quien la dota de sentido). Es cierto no obstante, según la observación del trabajo en el Taller, que la película escogida es también significativa para el participante y suele representar contenidos relativos a la propia historia personal, ya sea porque contenga elementos biográficos similares o por identificación con algún personaje principal de la película en cuestión, encontrándose referentes comunes en el desarrollo del proceso de identificación de constructos a lo largo del taller en otros ejercicios de videoterapia. Este dato apunta a que la elección de la secuencia preferida contiene de manera no consciente elementos de identidad personal profundos, o de "resonancia personal", utilizando el concepto de Larsen y Lazlo (Igartua, 2007).

Por otro lado, es interesante observar que en el trabajo de campo que se expone más adelante, varios participantes coincidieron en la elección de su secuencia y de entre todo el universo cinematográfico posible, tres eligieron la secuencia de $E l$ piano en la que la protagonista se ata a su piano cayendo ambos al mar; otras dos eligieron la secuencia final de Lo que el viento se llevó. Podría revelar esta circunstancia que ¿quizás haya secuencias del cine que tienen un mayor impacto para el código colectivo, teniendo en cuenta además que el año de producción de ambas es muy distante (1993 y 1939 respectivamente) y que no existe correlación entre el año de producción y edad del participante?

Pasamos pues a describir la metodología y el procedimiento de esta propuesta. Antes decir, que más allá del ámbito en el que nace este estudio (la consulta de terapia privada), esta técnica es extensible a cualquier ámbito de la salud mental, la educación o la intervención en colectivos sociales. Y aunque se presenta en formato grupal, se han realizado por parte de la investigadora, algunas experiencias en tera-

\footnotetext{
${ }^{7}$ R.C. Sánchez en Montaje cinematográfico. Arte de movimiento (pg. 53), define la secuencia como una división del film que posee un sentido completo y la escena como una acción continuada, filmada en un mismo ambiente o escenario y que carece de sentido completo.
} 
pia individual con resultados muy favorables. Igualmente se han planteado algunas experiencias sin soporte audiovisual con colectivos, siendo interesante la posibilidad de generar sentido, aún sin el visionado del material fílmico.

\section{MÉTODO}

El tipo de metodología utilizado es descriptiva, de orientación cualitativa y de observación abierta.

Para el posterior análisis, se realizó una revisión del material fílmico seleccionado por los participantes y se elaboró una pequeña entrevista voluntaria de tres preguntas, con el propósito de generar información narrativa subjetiva complementaria a la experiencia, siendo las respuestas publicadas en un blog anónimo y privado a fin de poder visualizar el texto junto a la secuencia elegida. Finalmente se estudiaron las respuestas.

\section{ESTRATEGIAS METODOLÓGICAS}

El objetivo general es indagar cómo una pieza fílmica aislada, puede ser un elemento de elicitación de constructos personales para la elaboración de la narrativa propia en relación con la identidad. El objeto de este estudio es la construcción del autoconcepto en clientes voluntarios y el campo del mismo "la secuencia cinematográfica" como herramienta terapéutica desde una perspectiva constructivista (Neimeyer y Mahoney, 1998).

Por ello, utilizamos una variedad de métodos a fin de poder desglosar los ítems de interés para la investigación, en concordancia con el análisis epistemológico de la investigación biográfico-narrativa propuesto por Bolívar (2002). En línea con las propuestas de la Investigación Basada en las Artes (Hernández, 2008) sería necesario, para una mayor comprensión de este estudio, completar la lectura de este artículo con el visionado del blog de la secuencia elegida por el participante, junto a sus explicaciones personales sobre lo que significa para él su elección. Aquí, sólo se transcribirán algunos pequeños párrafos del texto para apoyar la exposición. El lector interesado puede contactar con la autora para cursar la invitación privada al contenido de dicho blog.

Las premisas de las que parte la investigadora son, que cada individuo posee la llave de su significado identitario como ser y que se manifiesta en el material que elige como significativo. Indagar el mundo interno implica tratar con el relato construido sobre sí mismo. Elegir la secuencia que más nos ha llegado de las películas que hemos visto, es ya un acto significativo que está relacionado con nuestro mundo interno. Los elementos que forman la pieza narrativa no nos son ajenos ni casuales. Voderer distingue entre un modelo analítico y un modelo implicado de recepción de la ficción mediática, siendo la diferencia entre ambos el grado de implicación emocional (Igartua, 2007). Nuestro supuesto es que elegimos una secuencia entre todas las posibles, desde la identificación o desde la idealización, pero siempre desde una implicación emocional o autobiográfica por resonancia con 
nuestro autoconcepto. Es proyección de una parte de sí, en la que partes del mundo interno son rescatadas mediante la simbolización del relato o de sus elementos. Habla de nosotros y habla por nosotros con una elocuencia difícilmente superable.

La pregunta central generalizada sería entonces: ¿Ese significado personal se desvela en cada elección del material artístico que cada individuo escoge como "preferido"?

\section{POBLACIÓN Y MUESTRA}

Las fuentes de información para elaborar el estudio fueron los sujetos participantes en el Taller de Videoterapia Experimental, población general convocada mediante publicidad en todo el territorio español. Participaron en el taller un total de 83 personas desde 2005 hasta junio de 2010, fecha de finalización de este estudio, siendo aplicada la TSC a 61 personas. Para el estudio se descartaron aquellos participantes que no reunieron criterios idénticos de situación por no realizar la TSC en set de trabajo de espaciointerno y aquellos que no respondieron a la entrevista (por no poder contactar o porque declinaron realizarla), resultando 37 sujetos válidos (tabla 1).

\section{Instrumentos de recogida de información}

Se utilizaron los siguientes instrumentos:

- Se aplicó la TSC

- Anotaciones de la terapeuta-investigadora entre 2005-2010.

- Se realizó una revisión del material fílmico seleccionado retrospectivamente en el caso de participantes que ya habían realizado el taller (antes de mayo 2009)

- Recopilación prospectiva del material seleccionado por los participantes a partir de esta fecha.

- Entrevista voluntaria de tres preguntas, con objeto de generar información subjetiva que esclareciera la experiencia de los participantes.

- Blog anónimo y privado creado a fin de poder visualizar el texto de las respuestas a la entrevista, que fueron publicadas junto a la secuencia elegida.

\section{TÉCNICA DE LA SECUENCIA DE CINE (TSC)}

Se aplicó la TSC con el siguiente esquema de trabajo en los distintos encuentros del Taller de Videoterapia Experimental.

$\checkmark \quad$ Elección de una secuencia cinematográfica por parte del participante.

$\checkmark \quad$ Visionado en grupo de la misma

$\checkmark$ Elaboración del significado personal asociado a la elección.

$\checkmark \quad$ Integración de polaridades de constructos mediante técnicas psicocorporales gestáltistas. 
LA TSC se realiza en dos encuentros. En el primer encuentro se encarga la tarea de la búsqueda de una secuencia con la consigna siguiente:

Tarea $\mathrm{n}^{\circ} 1$ : "Piensa en una secuencia de película de duración aproximada a dos minutos que sea representativa o significativa para ti. Tráela en DVD para el próximo taller"

No se ofrecen muchas más explicaciones. La terapeuta suele añadir si le demandan más información: "la que te venga a la cabeza".

En el segundo encuentro, más de quince días después, la terapeuta invita a los asistentes a mostrar su secuencia. Antes del visionado se realiza la siguiente pregunta:

Pregunta ${ }^{\circ} 1$ ¿ ¿Por qué has elegido esta secuencia y no otra?

Posteriormente se procede al visionado, realizando al final del mismo la pregunta $n^{\circ} 2$.

Pregunta $\mathrm{n}^{\circ} 2:$ ¿Qué dice de ti esta secuencia?

Si el participante lo desea recibe un feedback de los compañeros una vez terminada su reflexión. Al final de los visionados de todos los participantes, cada uno de ellos responde a la última pregunta:

Pregunta nº3: ¿Qué parte de ti muestra esta secuencia?

Después trabajamos con la definición e integración de polaridades mediante ejercicios psicocorporales de orientación gestaltista.

\section{ANOTACIONES DE LA TERAPEUTA-INVESTIGADORA}

Las anotaciones de la terapeuta-investigadora entre 2005-2010 son fuente de datos subjetivos que han aportado preguntas al presente estudio y que han ido añadiendo datos para acotar la forma de aplicación de la TSC. Rescataremos aquí algunas observaciones de la terapeuta-investigadora que pueden ser de utilidad para comprender lo dicho.

En la ronda de inicio del segundo encuentro, algunas personas muestran su dificultad para elegir una secuencia.

Una participante explicó que suponía que los demás traerían unas secuencias de culto y ella no se atrevía a traer la suya por considerarla ñoña. 
Algunos traen dos para optar según las escogidas por los otros. Otros participantes, la eligen pero no la traen, alegan que por dificultad técnica, que no se han vuelto a acordar $u$ otras razones.

Como terapeuta apoyo el trabajo con la secuencia elegida primigeniamente, aunque sea sin soporte audiovisual para el visionado grupal. En vista de que esto pasaba en algunas ocasiones y siendo consciente de que el trabajo pierde en fuerza expresiva y terapéutica, desde los dos últimos talleres he empezado a utilizar la búsqueda en you-tube o similar el mismo día del taller, para los participantes que no hubieran traído DVD o la secuencia elegida primigeniamente. Me sorprende que siempre esté disponible en la red.

Habitualmente se prolonga el silencio hasta que uno decide mostrar su película. Por el modo en que se suele producir este acontecimiento, interpreto que hay una conciencia subyacente de que se muestra algo muy personal.

Normalmente la respuesta a ¿por qué has elegido esta secuencia y no otra?, viene a ser bastante general, por ejemplo: "siempre me ha gustado", "la he visto cientos de veces", "no sé por qué pero me emociona siempre que la veo". No suele haber a priori una conciencia clara de que uno está contando algo personal a través de su elección, quizás hay una cierta sospecha.

Una vez visionada suele hacerse un silencio conmovedor. La expresividad de la narración cinematográfica asociada a la persona que la muestra es muy impactante. Mi sensación cada vez que esto se produce es de sobrecogimiento porque siento que se desvela el mundo no expresado del ser que está al lado. Es más amplio lo expresado que el contenido.

La respuesta a ¿qué dice de ti esta secuencia? es para mí como terapeuta un momento de revelación personal, un acto de transparencia. Es una posibilidad de reapropiarse de lo proyectado

A continuación algunas anotaciones sobre algunas TSCs. Entre paréntesis el posible eneatipo de personalidad que asigna la terapeuta al participante, a continuación el título de la película elegida, una frase rescatada por la terapeuta como significativa en la exposición del participante y finalmente la polaridad trabajada.

- (5) UN LUGAR EN EL MUNDO Al límite COBARDE VS OSADO

- (4) EL PIANO ¿Ha decidido mi voluntad la vida? SUFRIDORA VS VIVA

- (4) CUENTA CONMIGO Compartir la alegría PENITENTE vs ALEGRE 
- (2) JESUSCRISTO SUPERSTAR El sacrificio EL HIJO VS EL HOMBRE

- (4) CARICIAS. Romper con lo políticamente correcto. INSOLENTE VS TIMIDO

- (3) PEQUEÑA MISS SUNSHINE. La niña que necesita. NO CUENTO VS CENTRO ATENCION

- (6) AMOR INMORTAL. El engaño SEGURO VS DESCONFIADO

- (9) ALGUIEN VOLO SOBRE EL NIDO DEL CUCO. La liberación... INDOLENTE VS RABIOSA

- (4) AMERICAN BEAUTY. Sacar las emociones VERGONZOSO VS QUE ME EXPRESO

- (2) BARRY LYNDON El juego "para que me quieran". SEDUCTOR VS NO VALIDO

- (5) NOVIEMBRE DULCE La vida afuera OBSERVADORA VS VIVIENDO

- $\quad(5 / 6)$ DESEANDO AMAR. El secreto HIPOCRITA VS SINCERO

- (9) CON FALDAS Y A LOCO. Nadie es perfecto CONFORMISTA VS INFLEXIBLE

- (9) LA VIDA ES BELLA. La rebeldía CONCILIADOR VS INTRANSIGENTE

- (6) ESPLENDOR EN LA HIERBA. La locura y la verdad SOMETIDA VS REBELDE

- (2) FLASHDANCE. El examen COMPLACIENTE VS LIBRE

- (3) LO QUE EL VIENTO SE LLEVÓ. La manipulación de los ... MANIPULADORA VS AUTENTICA

- (8) LA ROSA PURPURA DEL CAIRO. Dentro y fuera de la pe... INGENUA VS DURA

- (7) HEAT. La flexibilidad DESCONFIADO VS CONFIADO

- (6) LA VIDA EN ROSA - La conexión con la vida FUERTE VS TIERNA

- (9) LO QUE EL VIENTO SE LLEVÓ. Me volvería loca EVITADORA VS CONFRONTADORA

- (5) GREASE. Soltarse la melena ABURRIDO VS GAMBERRO

- (6) EL PIANO. Tirando el piano al mar KAMIKAZE VS AUTOCUIDADO

- (8) MEJOR IMPOSIBLE. Pisando rayas en las aceras LOCA VS NIÑA

\section{ENTREVISTA}

Se realizó a posteriori de la aplicación de la TSC con el objetivo de recoger significados personales asociados a la misma. Se trata de una entrevista voluntaria de tres preguntas con respuesta abierta: 1. - ¿Qué secuencia y qué película elegiste?; 2.- ¿Qué dice de ti esta secuencia?; 3.- ¿Qué te ha aportado a tu vida el trabajarla en el taller?

Se intentó contactar con aquellos participantes que habían realizado el taller anteriormente a mayo de 2009 y se propuso a los participantes que lo realizaron des- 
pués. Se informó a todos de la voluntariedad de su respuesta y de que se trataba de una investigación para el doctorado de la terapeuta, publicándose las respuestas junto a su secuencia en un blog con tratamiento anónimo y de acceso privado, al que tendrían acceso una vez recibidas sus respuestas.

\section{BLOG}

Elaboración de un blog anónimo y privado para muestra y análisis del material. En el blog se pueden ver algunas muestras de lo aquí explicado. Transcribimos un ejemplo:

¿Qué secuencia y qué película elegiste? Pequeña Miss Sunshine. Después de la llamada de que la niña es aceptada en el concurso y ella ajena a los problemas que estaba teniendo la familia comienza a gritar por toda la casa... ¿Qué te ha aportado el trabajarla en el taller? Al mostrar esta película a los demás y explicar el por qué de la película, me hizo y me hace tomar consciencia, de que llevo una niña dentro que pide atención y que cuando no la tiene se frustra y piensa... o mejor pienso, que es por algo que hago mal, porque no cae bien, porque no gusta, etc. Lo más importante es que me di cuenta y me doy, que aunque llevo a esa niña dentro, las cosas no las puedo mirar solo desde sus ojos sino, que el prisma es más grande.

\section{PROCEDIMIENTO}

Se partió de un material previo, en el que ya la TSC se había aplicado, concretamente desde el segundo taller realizado en febrero de $2006^{8}$.

Podemos definir varias etapas en esta investigación:

- $1^{\mathrm{a}}$ etapa. Utilización de la TSC sin integración gestalt y corporal

(Grupos 03/06; 01/07; 09/07) Se caracteriza por ser un periodo tentativo. Se hacen las primeras observaciones sobre cómo plantear la sistematización de la propuesta de trabajo para buscar mayor eficacia terapéutica del mismo.

- $2^{\mathrm{a}}$ etapa: Utilización de la TSC con integración gestalt y corporal

(Grupos 10/07 en adelante) La segunda etapa que se inicia con el grupo del 10/07 se caracteriza por introducir una modalidad estructurada de trabajo para elaborar los constructos asociados al visionado de la secuencia elegida, definiendo sus polaridades y trabajando después con la integración mediante técnicas de terapia Gestalt

- $\quad 3^{\text {a }}$ etapa: Cuestionario y creación del blog. Recogida de datos general con antiguos participantes voluntarios.

\footnotetext{
${ }^{8}$ En el primero no fue posible porque participaron 22 personas y habría sido muy laborioso aplicarlo con un grupo tan numeroso
} 
(Mayo 2009) La tercera etapa viene condicionada por la necesidad de recogida de datos. Se realiza una entrevista de tres preguntas cortas que faciliten la participación en la misma. Se contacta por correo electrónico con todos los antiguos participantes, salvo con 7 con los que se ha perdido el contacto en este tiempo. En mayo de 2009 se crea un blog anónimo y privado en el que se exhiben las respuestas sin modificar, junto a la secuencia escogida por el entrevistado para poder visionarla. Se invita después al entrevistado para que pueda acceder al blog cuando quiera.

- $4^{\mathrm{a}}$ etapa: Utilización de la TSC con integración gestalt y corporal y cuestionario para el blog con nuevos participantes.

(Grupos 01/08; 09/08; 09/09; 01/10; 03/10) La cuarta etapa comienza con los grupos que han realizado el taller después de la creación del blog. A estos grupos se les presenta la entrevista en la semana siguiente al trabajo y realizan la respuesta voluntaria sin acotación del tiempo.

- $5^{\text {a }}$ etapa: Análisis y elaboración del presente estudio.

- $6^{a}$ etapa: Utilización de la TSC con integración gestalt y corporal y cuestionario para el blog con nuevos participantes.

La sexta etapa corresponde a la investigación estructurada en curso, sobre cineterapia y videoterapia. Grabación de las respuestas in situ, registro y análisis de las polaridades de constructos, relaciones entre constelación semántica y autoconcepto, etc.

\section{RESULTADOS}

A través de las respuestas a la entrevista que se realizó a los participantes podemos rastrear algunos datos:

- ¿Puede el participante explicar un significado personal asociado a la secuencia por medio de pronombres reflexivos?

- ¿Existe alguna referencia en las respuestas que den evidencia de que el trabajo ha aportado algún darse cuenta al participante?

- ¿Se da muestra de utilidad para la autoestima en las respuestas?

- ¿Existen señalamientos por parte del paciente hacia los otros componentes del grupo en su relato?

- ¿Qué tipo de película es escogida por los/las participantes en función del género del film, de la edad, del sexo, la nacionalidad de la producción?

- ¿Existe correlación entre tipo de secuencia y estilo cognitivo?

- ¿Existe correlación entre tipo de secuencia y trastornos de la personalidad?

Los datos agrupados pueden verse en la siguiente tabla: TABLA 1.- Resumen de datos 


\begin{tabular}{|c|c|c|c|c|c|c|c|c|c|c|}
\hline 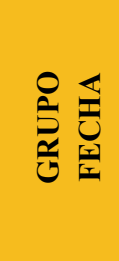 & 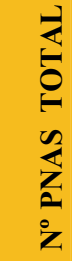 & 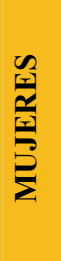 & $\sum_{\substack{n \\
0}}^{\infty}$ & 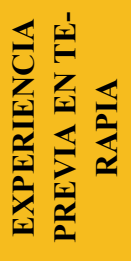 & 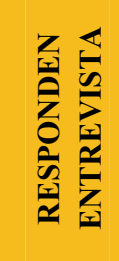 & 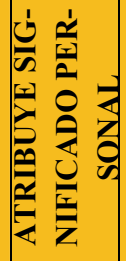 & 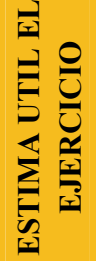 & 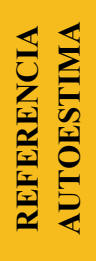 & 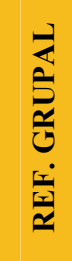 & $\sum_{\substack{\mid c \\
1}}^{\infty}$ \\
\hline $03 / 06$ & 6 & 4 & 2 & 6 & 5 & 5 & 4 & 4 & 0 & $1^{\mathrm{a}}$ \\
\hline $01 / 07$ & 8 & 6 & 2 & 6 & 5 & 3 & 3 & 2 & 0 & $1^{\mathrm{a}}$ \\
\hline $09 / 07$ & 5 & 3 & 2 & 3 & 3 & 2 & 2 & 2 & 0 & $1^{\circ}$ \\
\hline $10 / 07$ & 7 & 4 & 3 & 0 & 2 & 2 & 1 & 1 & 0 & $2^{\circ}$ \\
\hline $01 / 08$ & 6 & 3 & 3 & 5 & $6 *$ & 6 & $4 * *$ & $3^{*}$ & 3 & $3^{\mathrm{a}}$ \\
\hline $09 / 08$ & 6 & 3 & 3 & 4 & 6 & 4 & 3 & 4 & 4 & $3^{\circ}$ \\
\hline $09 / 09$ & 5 & 2 & 3 & 4 & 5 & 5 & 5 & 3 & 2 & $4^{\circ}$ \\
\hline $01 / 10$ & 6 & 3 & 3 & 5 & 5 & 4 & 5 & 3 & 1 & $4^{\circ}$ \\
\hline $03 / 10$ & 12 & 12 & 0 & ¿? & (4) & - & - & - & - & $4^{\circ}$ \\
\hline Total & 61 & 40 & 21 & 33 & 37 & 31 & 29 & 20 & 10 & \\
\hline
\end{tabular}

De las 37 personas que respondieron a la entrevista, 6 no pudieron explicar un significado personal asociado a la secuencia (las 6 evitan el pronombre reflexivo; 1 hace referencia a las emociones que le aporta como espectadora, 4 le atribuyen un significado universal, 1 describe lo que ocurre). Veamos algunos ejemplos transcritos del blog:

Ejemplo de $N O$ apropiación del significado: ¿Qué secuencia y qué película elegiste? Secuencia del Fever club de Collateral, de Michael Mann ¿Qué dice de ti esta secuencia? Que hay gente muy malvada dispuesta a cualquier cosa por conseguir su objetivo o por dinero, sin importarles nada los demás incluso la vida.

Ejemplo de apropiación del significado: ¿Qué secuencia y qué película elegiste? secuencia final del accidente en "El sexto sentido" ¿Qué dice de ti esta secuencia? Dice que he tenido que superar mis terribles miedos por mi mismo desde muy niño.

De las 37 personas que respondieron a la entrevista, 29 hicieron referencia a que les había sido útil con términos como: Ver que (3), darse cuenta de (5), comprobar que (1), servido para (2), conciencia de (8), entender que (2), que necesito (1), nueva mirada (1), integrar mi (1), conectar con (1) (En la tabla $\mathrm{n}^{\circ} 1$ de datos : * 1 respondió a la utilidad del taller en general, ${ }^{* *} 1$ respondió al taller y a la secuencia). 
Ejemplo: ¿Qué secuencia y qué película elegiste? Irma la dulce. Cuando ella baila encima de una mesa de billar. ¿Qué te ha aportado el trabajarla en el taller?: Me di cuenta de que no quiero apoderarme de la fuerza que tengo y me ha ayudado a hacerlo, voy asimilándolo y me hago cargo del camino que tengo que recorrer entre mis dos extremos, estar apoderada y sentirme como una mierda..."

Ejemplo: ¿Qué secuencia y qué película elegiste? Esplendor en la hierba, cuando la chica está en el baño ¿Qué te ha aportado el trabajarla en el taller? La escena en sí fue un elemento que me ayudó a integrar lo vivido durante el taller, desnuda frente a la cámara, con mi cuerpo, mis emociones y mis pensamientos. Una forma de salir de la locura de no asumirme con todo lo que soy. Todavía hoy no puedo encontrar las palabras que pongan conciencia a lo que siento cuando presencio esta escena. Es la imagen en si misma la que condensa todos los matices que hace que pueda digerir un dolor que no puedo poner en palabras. Para mi se ha convertido en una herramienta de conciencia"

8 no expresan directamente utilidad del ejercicio para sus vidas (1 directamente no respondió a esta pregunta, 1 expresa vergüenza de lo mostrado y le llevó a hacer terapia individual, 1 algo que ya sabía, 1 aprendió de las de sus compañeros, 2 les hace recordar, 2 no hacen referencia)

Ejemplo: ¿Qué secuencia y qué película elegiste? Se trata de la película "The inmortal beloved". La secuencia correspondía con la primera parte de la película y es esa en la que un duque y su hija engañan a Beethoven para que toque el piano y demostrar así que no es incapaz como se rumorea. ¿Qué te ha aportado el trabajarla en el taller? ...lo que me ha aportado ha sido más enfocado a mi percepción del mundo que sobre mi mismo. Y lo que he aprendido de mi mismo ha sido a través de las explicaciones de mis compañeros sobre sus propios videos.

De las 36 que respondieron a la tercera pregunta, 20 hacen referencia en términos de autoestima: apoderándome, reubicar la experiencia, me importa la vida, abierto a, aceptación de, retomar mi, verme guapa, al fondo serenidad, alegría de vivir, poder expresar, el prisma más grande, cosas lindas, la vida hermosa, persistiré, aún me sirve, más relajado, reapropiarme, arriesgarme, necesidad de salir del dolor, recuperar mi niña.

Ejemplo: ¿Qué secuencia y qué película elegiste? Peor Imposible. Jugando u obsesionado en no pisar las rayas de las aceras. ¿Qué te ha aportado el trabajarla en el taller? "Lo que me ha aportado esta escena e incluso la evolución de curso de videoterapia es que tras años de buscar algo, 
ver lo duro de pelar que ha sido mi proceso, ha sido como por fin encontrarme y tener presente a mi niña/o interna/o..."

De las 36 que respondieron a la tercera pregunta, 10 hacen algún señalamiento a la experiencia grupal con referencias como: los compañeros (5), escucha de los otros (1), los demás (1), poner en común (1), la gente (1), otros veíais (1).

Ejemplo: ¿Qué secuencia y qué película elegiste? Elegí la escena final de la película La vida de Brian, cuando cantan todos los personajes crucificados "Always looks at the bright side of life" (Mira siempre el lado bueno-amable de la vida) ¿Qué te ha aportado a tu vida el trabajarla en el taller? Me ha gustado mucho trabajarla en el taller. Me ha sorprendido porque iba con la idea de haber elegido "mal... Luego me di cuenta de que era estupenda y era la mejor elección. El tema sempiterno: la muerte (= inseguridad de lo seguro) y tantas cosas que me evoca...UF! no me apetecía. Me sorprendió y agradó que, primero escuchando las risas de mis companeros, luego acabara tan a gusto riendo yo misma. Nada, debería ser tan tremendo como para no llevarlo bien, incluso con sentido del humor, porque depende de nuestro propio enfoque de las cosas como éstas nos afecten. Me he sentido acompañada y agradecida por ello.

Es interesante decir aquí, que en los cuatro primeros grupos no hay referencias al resto de participantes. Sí aparecen a partir de introducir el trabajo de integración corporal, no siendo posible concluir si el cambio en los resultados puede correlacionar con el cambio de propuesta o con cambios en la intervención, dada la condición de investigadora-terapeuta y por lo tanto "observadora participante"

TABLA 2.- Ordenada por sexo, edad y nacionalidad de producción

\begin{tabular}{|l|l|l|l|l|}
\hline SEXO & EDAD & NAC. PROD & GENERO & AÑO \\
\hline V & $20-30$ & EEUU Náufrago & Aventura.drama & 2000 \\
\hline V & $20-30$ & EEUU American Beauty & Drama & 1999 \\
\hline V & $20-30$ & EEUU Amor inmortal & Drama & 1994 \\
\hline V & $20-30$ & Hong Kong, Francia, Thai. Deseando amar & Drama.romántic & 2000 \\
\hline V & $20-30$ & UK-EEUU Atrapado en el tiempo & Comedia.románt & 1993 \\
\hline V & $30-40$ & Argentina Un lugar en el mundo & Drama & 1992 \\
\hline V & $30-40$ & EEUU Heat & Acción & 1995 \\
\hline V & $30-40$ & EEUU Persiguiendo sueños & Drama & 2000 \\
\hline V & $30-40$ & EEUU Jesuscristo superstar & Musical & 1973 \\
\hline V & $30-40$ & España Caricias & Drama & 1997 \\
\hline V & $30-40$ & España, Argentina En la ciudad sin limites & Drama.suspens & 2002 \\
\hline V & $40-50$ & EEUU Collateral & Acción.supense & 2004 \\
\hline V & $40-50$ & EEUU Pi (fe en el caos) & Intriga & 1998 \\
\hline
\end{tabular}




\begin{tabular}{|l|l|l|l|l|}
\hline V & $40-50$ & EEUU Grease & Musical & 1978 \\
\hline V & $40-50$ & EEUU El sexto sentido & Suspense & 1999 \\
\hline V & $40-50$ & Italia La vida es bella & Comedia.drama & 1997 \\
\hline V & $50-60$ & UK Barry Lyndon & Drama & 1975 \\
\hline M & $20-30$ & EEUU Pequeña Miss Sunshine & Comedia.drama & 2006 \\
\hline M & $20-30$ & EEUU Nell & Drama & 1994 \\
\hline M & $20-30$ & EEUU Esplendor en la hierba & Drama.romántic & 1961 \\
\hline M & $20-30$ & EEUU Lo que el viento se llevó & Drama.romantic & 1939 \\
\hline M & $20-30$ & EEUU Flashdance & Musical & 1983 \\
\hline M & $30-40$ & EEUU Irma la dulce & Comedia & 1963 \\
\hline M & $30-40$ & EEUU Cuenta conmigo & Drama.aventur & 1986 \\
\hline M & $30-40$ & EEUU Noviembre dulce & Drama romántic & 2001 \\
\hline M & $40-50$ & Australia, Nueva Zelanda, Francia El piano & Drama & 1993 \\
\hline M & $40-50$ & Australia, Nueva Zelanda, Francia El piano & Drama & 1993 \\
\hline M & $40-50$ & EEUU Kunfu panda & aventuras & 2008 \\
\hline M & $40-50$ & EEUU La rosa purpura del Cairo & Comedia.románt & 1985 \\
\hline M & $40-50$ & EEUU Mejor imposible & Comedia.románt & 1997 \\
\hline M & $40-50$ & EEUU Alguien voló sobre el nido del cuco & Drama & 1979 \\
\hline M & $40-50$ & EEUU Memorias de África & Drama.romántic & 1985 \\
\hline M & $40-50$ & España Mujeres al borde de un ataque de ... & Drama comedia & 1988 \\
\hline M & $40-50$ & Francia La vida en rosa & Drama & 2007 \\
\hline M & $40-50$ & UK La vida de Brian & Comedia & 1979 \\
\hline M & $50-60$ & EEUU Con faldas y a loco & Comedia & 1959 \\
\hline M & $50-60$ & EEUU Lo que el viento se llevó & Drama.romántic & 1939 \\
\hline M & $50-60$ & Francia La fortuna de vivir & Comedia drama & 1998 \\
\hline Total & & 25 EEUU & 20 drama & \\
\hline 37 & & 12 otros países & 6 comedia & \\
\hline & & & 3 musció/aven/susp & \\
\hline & & & & \\
\hline
\end{tabular}

De los participantes válidos para el estudio, 25 escogieron películas estadounidenses, 2 de las cuales son de cine independiente fuera del gran cine comercial. El resto de nacionalidades de producción son 3 UK, 1 Argentina, 2 Australia y otros, 3 España, 2 Francia, 1 Hong Kong y otros, 1 Italia.

Respecto a la preferencia por géneros (para categorizar el género se utilizaron datos coincidentes en dos buscadores - filmaffinity.com /dvdenlared.com - estimando el criterio coincidente y un segundo caracterizador), atendiendo al primer caracterizador de género cinematográfico, se observó que: de los 6 que eligieron "acciónsuspense-aventuras-intriga" 5 fueron hombres; 20 personas prefirieron "drama" (6 románticos); 9 "comedia" (como segundo caracterizador: 3 drama y 3 románticas), y 3 musical. 
Sobre los años de producción 5 de 1930-1970, 5 década 70, 5 década 80, 14 década 90, 9 década 10, no observando por conteo simple correlación aparente entre edad del participante y el año de producción (ver tabla 2)

\section{DISCUSIÓN Y CONCLUSIONES}

No es posible con el presente estudio responder adecuadamente a la pregunta central de si ¿el significado personal se desvela en cada elección del material artístico que cada individuo escoge como "preferido"? Se puede inferir, no obstante, de los datos aportados por los participantes en sus respuestas a la entrevista, que la TSC podría ser apropiada para elicitar constructos relativos al autoconcepto personal, facilitando una mayor conciencia del relato propio sobre la identidad del sujeto, ya que se observa con frecuencia en las respuestas, una reapropiación de la narrativa fílmica exógena como elemento evocador autorreferente de los constructos personales identificados en la secuencia.

En la mayoría de los casos se contesta que fue de utilidad terapéutica, con emisiones verbales en relación a la toma de conciencia de la problemática personal y la necesidad de cambio.

Igualmente se realizan abundantes referencias espontáneas en torno a la importancia de mostrar/mostrarse/compartir en grupo este trabajo, como liberador y empático.

Sorprende la mayoría abrumadora de películas de nacionalidad estadounidense escogidas por los participantes. Podría decirse que, en este estudio, película es sinónimo de cine/EEUU. Sugiere este hecho una pregunta ¿de qué manera pertenece nuestro código figurativo colectivo a la cultura norteamericana? Es interesante apuntar el impacto que la industria cinematográfica norteamericana pueda tener en el proceso de construcción de la identidad en nuestro país.

Se observan algunas consistencias en cuanto a la elección del género cinematográfico de acción/suspense/intriga que fue mayoritariamente elegido por hombres. El género musical, fue altamente preferido por participantes con la asignación de rasgo de personalidad histriónica por parte de la terapeuta.

Aunque no se aportan datos en esta publicación, la terapeuta observó la posible relación entre la extensión de respuesta a la entrevista y psicopatologías diagnósticas de trastornos graves de la personalidad, declaradas por los participantes en el transcurso del taller.

Según posibles estudios más sistemáticos basados en la replicabilidad, la intervención de la terapeuta es una variable a controlar debido a la dinámica propia de la experiencia en la que la adscripción a un modelo u otro de intervención (fenomenológica, conductual, etc) podría tener efectos sobre el resultado.

Finalizando y en términos de análisis de los datos, este estudio puede servir para elaborar categorías exhaustivas y excluyentes que permitan aumentar la fiabilidad interjueces con vistas a siguientes fases de investigación. Este estudio descriptivo 
de la aplicación de la TSC, puede entenderse a ese efecto, como una tentativa de organizar los datos adquiridos. Sirviendo así, como guía para desarrollar nuevos diseños en entornos más consistentes y homogéneos, que permitan un mayor control del estudio y facilitar la labor estadística y la generalización de resultados.

\section{REFERENCIAS BIBLIOGRÁFICAS}

BOTELLA, L., FEIXAS, G. (1998) Teoría de los constructos personales, Barcelona, Editorial Laertes.

BOLÍVAR, A. (2002). “¿De nobis ipsis silemus?”: Epistemología de la investigación biográfico-narrativa en educación. Revista Electrónica de Investigación Educativa, 4 (1). Consultado el 08/02/2011. http://redie.uabc.uabc.mx/vol4no1/contenido-bolivar.html

DEBRAY, R. (1994) Vida y muerte de la imagen, Barcelona, Ediciones Paidós

FERNÁNDEZ-BALLESTEROS, R. (2001) Introducción a la evaluación psicológica, Vol. I, Madrid, Ediciones Pirámide, pp 279-346

GIROUX, H. A. (2003) La inocencia robada. Juventud, multinacionales y política cultural, Madrid, Ediciones Morata S.L.

HERNÁNDEZ, F. (2008) "La investigación basada en las artes. Propuestas para repensar la investigación en educación", Educatio Siglo XXI, n. ${ }^{\circ} 26 \cdot 2008$, pp. $85-118$

IGARTUA J. (2007) Persuasión narrativa: El papel de la identificación con los personajes a través de las culturas, Alicante, Editorial Club Universitario.

MAMPASO, A. (2001) "La video-animación: aplicaciones en los campos de desarrollo social y comunitario, la educación artística y el arte terapia". Tesis Doctoral. Universidad Complutense de Madrid.

MAMPASO, A., RUEDA, O., PÉREZ, I. Y MORALES, M. (2010) “Aplicaciones audiovisuales en terapia", Póster I Congreso Nacional de Arteterapia (FEAPA), Las voces de la profesión y sus campos de intervención, 26, 27 y 28 de Marzo. Girona.

NEIMEYER, R.A., MAHONEY, M.J. (1998) Constructivismo en psicoterapia, Barcelona, Paidós Ibérica. 